\title{
Study and Design of a Differentially-Fed Tapered Slot Antenna Array
}

\author{
Eloy de Lera Acedo, Student Member, IEEE, Enrique García, Member, IEEE, Vicente González-Posadas, \\ José Luis Vázquez-Roy, Rob Maaskant, and Daniel Segovia, Member, IEEE
}

\begin{abstract}
The results of a parametric study and design of an ultrawideband dual-polarized array of differentially-fed tapered slot antenna elements are presented. We examine arrays of bunny-ear antennas and discuss the capabilities and limitations of differential antenna technology. As we focus on radio astronomical applications, the absence of a balancing-feed circuit not only reduces the first-stage noise contribution associated to losses in the feed, but also leads to a cost reduction. Common-modes are supported by the antenna structure when a third conductor is present, such as a ground plane. We demonstrate that anomalies may occur in the differential-mode scan impedance. Knowledge of both types of scan impedances, differential and common mode, is required to properly design differential LNAs and to achieve optimal receiver sensitivity. A compromise solution is proposed based on the partial suppression of the undesired common-mode currents through a (low loss) balancing-dissipation technique. A fully steerable design up to $45^{\circ}$ in both principal planes is achieved.
\end{abstract}

Index Terms-Antenna array feeds, mutual coupling, phased arrays, radio astronomy.

\section{INTRODUCTION}

$\mathbf{T}$ APERED SLOT antenna (TSA) Arrays are of large interest for ultrawideband applications from the time they were introdnced by Lewis et al. [1], in particular, the widely employed exponentially tapered slot antennas (Vivaldi antennas, [2]). In recent years, a growing interest has emerged from the radio astronomy community in the so-called aperture Arrays (AA) [3], which is the Enropean concept of a versatile array antenna composed of millions of dnal-polarized TSA elements. The SKA telescope [4] will comprise a nnmber of snch antenna array concepts, thereby facing one of the biggest technological challenges in radio astronomy for the 21 st centnry. In particnlar, the SKA telescope will have a collecting apertnre of one

Manuscripn received June 05, 2008; revised June 26. 2009. First published November 06. 2009; current version published January 04, 2010. This work was supported by the European Community Framework Programme 6. Sijuare Kiloneire Atray Design Studies (SKADS), under contract no. 011938 http:// www.skads-elloms.

E. de Leta Acedo was with the Astronomical Centre of Yebes, National Astrononnical Observatory of Spain. E-19080 Guadalajara, Spain. He is now with the Cavendish Laboralory. University of Canbridge, Canbridge CB3 OHE. U.K. (e-mail: eloy@mrao.cam.ac.uk).

E. García. J. Luis Vázquez-Roy, and D. Segovia are with the Signal Theory and Comınunicalions Departnent. University Carlos III of Madrid, Leganés. Matrid 28911, Spain (e-mail: legarcia@itsc.nc3m.es; jvazquez@tsc.uc3m.es; dani@isc.uc3m.es).

Vicente González-Posadas is with the Universidad Politécnica de Madrid. 28040 Madrid, Spain (e-mail: vgonzalz@euitt.upm.es).

Rob Maaskant is with the Netherlands Institute for Radio Astronony (ASTRON). 7990 AA Dwingeloo, The Netherlands (e-mail: maaskant (iasiron.nl),

Digital Object Identifier 10.1109/TAP.2009.2036193 square kilometer, and will cover a frequency band from 100 $\mathrm{MHz}$ up to $25 \mathrm{GHz}$ with dnal-linear polarization. The mid-frequency band, which ranges from 0.3 to $\mathrm{I} \mathrm{GHz}$, is intended to be covered by a dense array of millions of cheap TSA elements that can scan up to $\pm 45^{\circ}$. The array design represents a challenging problem for which different technologies are being considered [5]. This paper presents the benefits, the drawbacks, and a design example of a TSA array, which will be distinct from oher stndies published thus far [6]-[8] namely, the antennas are differentially-fed, thereby enabling us to use differential amplifiers and differential beam-forming technology in a straightforward manner.

Fig. 1 exemplifies five different types of TSA elements. Fig. 1(A) illnstrates an exponentially tapered aperture fed by a microstrip line printed on a relatively expensive and potentially lossy snbstrate. Such an antenna-feed transition acts as a balancing mechanism (balnn) for the differential TSA element, and is only required if a direct connection to a non-differential transmission line has to be realized [7]. Fig. I(B) represents a bilateral Vivaldi element composed of three metal layers [6]. Fig. 1(C) shows a differentially-fed nnilateral TSA element, however; it still requires a relatively expensive snbstrate. Fig. 1(D) illustrates a modified case compared to Fig. 1(A), where the metal sheet nsed for the exponential taper of the aperture is no longer printed on a dielectric snbstrate, but realized by relatively thick metallic plates that are composed of, e.g., alnminum [7]. Finally, an example of a differentially-fed TSA element, which is solely composed of thick metals, is shown in Fig. 1(E). This, so-called bnnny-ear antenna [9], which does not reqnire a (lossy) dielectric substrate or additional balancing feed board, represents an inexpensive and suitable candidate for the SKA project when aiming for differential technology. Obviously, the expensive and noisy feed board is no longer required if a low noise amplifier (LNA) is directly attached to the antenna and is realized in differential technology (coplanar strips for instance), or if the transition to single-ended technology has been realized within the LNA.

An important contribution to the design of differentially-ted array antennas that has been published by the SKA community thus far is the Australian "checkerboard" array [10], meant to be used as a focal plane array comprising a stack of a printed circuit board (PCB); a foam layer; and a ground plane. The PCB consists of an array of self-complementary rectangular conducting differentially-fed pair of patches with the appearance of a checkerboard printed on an electrically-thin dielectric substrate. The array is differentially-fed at the ground plane, with two-conductor transmission lines feeding the signals be- 


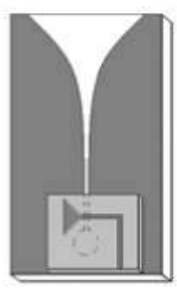

A

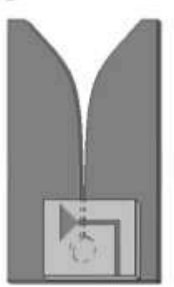

D

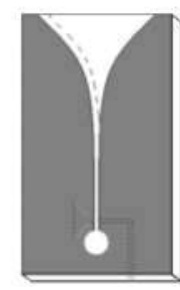

B

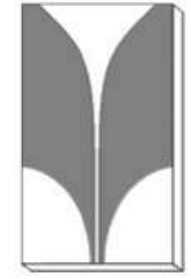

C

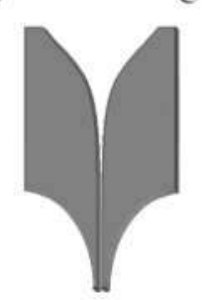

E

Fig. 1. Types of TSA elements: (A) Printed on a dielectric substrate with microstrip feed; (B) printed on a dielectric substrate with a stripline feed; (C) printed on a dielectric substrate with a differential feed; (D) one layer of thick metal with a localized microstrip feed, (E) one layer of relatively thick metals with a differential feed.

tween these points and the gaps between the corners of neighboring patches of the array [11]. The conducting surfaces of the connected-patch array are topologically equivalent to those of the tapered-slot array studied in this paper. This equivalence has been detailed in Section III. Another array concept, which has been fabricated and is currently being evaluated using integrated active receivers, is the dual-polarized array of differentially-fed tapered slot antennas with dual cavities [12]-[14]. This array has been printed on a thin and inexpensive polyester foil.

In this paper, arrays of dual-polarized differentially-fed bunny-ear antennas [Fig. 1(E)] are examined. In Section II, a parametric study of an infinite-by-infinite array of bunny-ear antennas is conducted, and used to analyze the effect of different antenna geometries on the scan impedance. The antenna elements are arranged on a square grid as shown in Fig. 2. By acquiring essential knowledge from previous papers [15], [16], and by using a commercial full-wave simulator based on finite elements method, named HFSS [17], it is now possible to design these complex structures and to analyze the vitally important mutual coupling effects as these yield the wideband performance of the array. The closely spaced elements exhibit strong mutual coupling effects which are still not modeled rigorously, so that full-wave simulations and design strategies are necessary [6]-[8].

The differential scan impedance anomalies are analyzed in Section III, which arise because of destructive mutual coupling interference effects. Generally, one can distinguish between several kinds of anomalies that may appear in the scan impedance [18]-[29]. A new type of impedance resonance for arrays of differentially-fed TSA elements has been identified and is shown to be similar to the resonance effect observed in [11]. This anomaly occurs due to the presence of a third conductor nearby the differential feeding line. This could represent the back metallic plane, which is used as a reflecting surface and a virtual ground for the differential feeding lines. Without precautions, a common-mode current may then be supported by the feed structure, and even

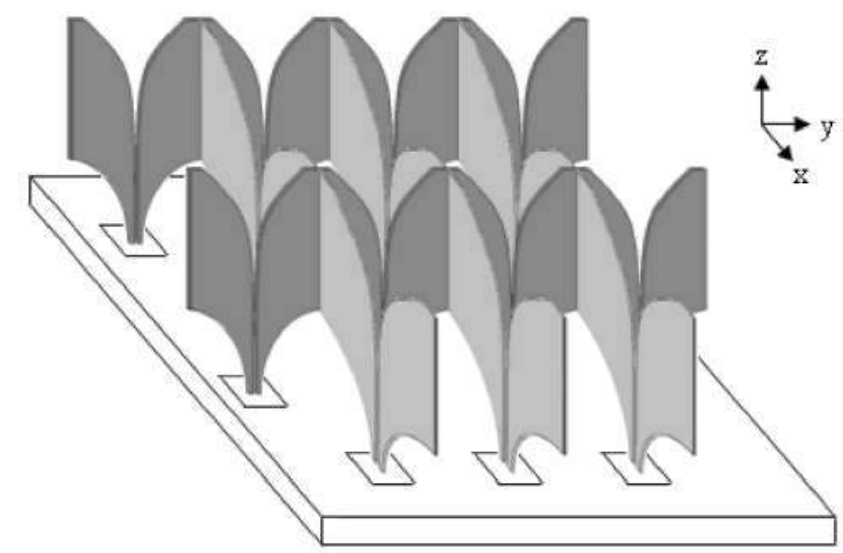

Fig. 2. Portion of a differentially-fed TSA element array with a metallic ground plane. One polarization is in dark gray and the other in light gray, the ground plane is in white. This represents a part of a larger array.

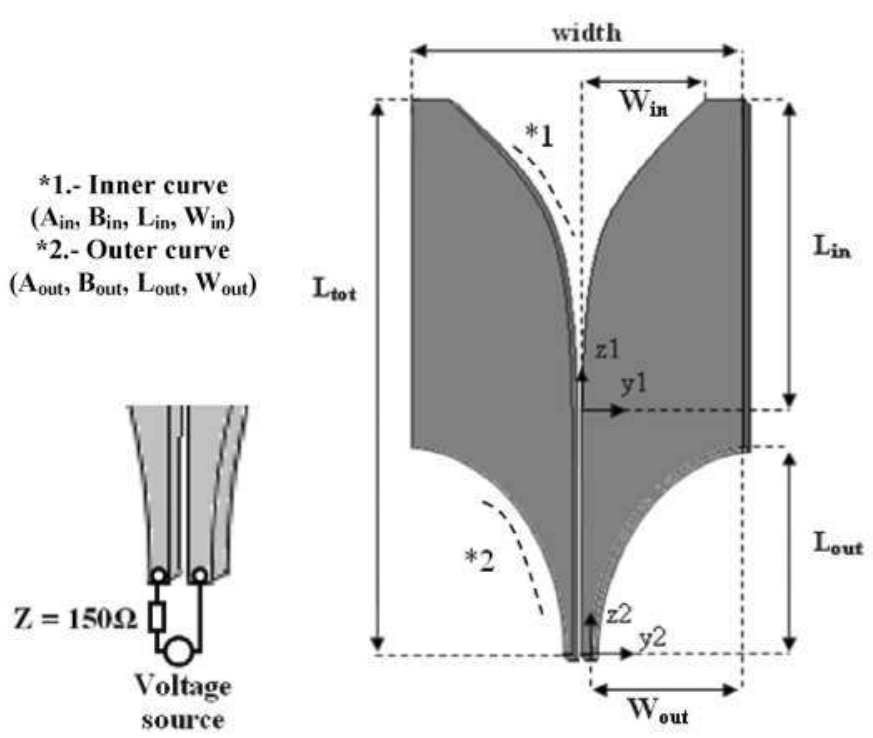

Fig. 3. Bunny-ear element geometry. The antenna is excited by a voltage source between the coplanar strips of the differential feed line at the bottom of the antenna as indicated in the figure (bottom left).

dominate over the differential-mode current for certain frequencies and array excitations/scan angles. As a result, a strong mismatch on the differential port is observed (see Fig. 3 for the port excitation setup), leading to a surge in the radiated power, and in turn, causes a scan blindness for the differential-mode to occur. We demonstrate that this impedance mismatch, which also leads to a noise mismatch in receiving array antennas, can be improved by means of a (low loss) balancing-dissipation technique, and we therefore study the resulting array noise figure as well.

In Section IV, a design example is presented of a dual-polarized array, which is steerable up to $45^{\circ}$ in both principal planes and operates over a frequency band ranging from $300 \mathrm{MHz}$ up to $1 \mathrm{GHz}$. Finally, the most relevant aspects of the study are summarized and the future work is presented.

\section{PARAmetric Study of The TSA Element}

The bunny-ear element in Fig. 3, inserted in an infinite-by-infinite array as in Fig. 2, is subject to a parametric analysis, i.e., 
the most important geometrical parameters of the antenna element are analyzed independently of each other. As indicated in Fig. 3, the antenna is excited by a voltage source which is attached to the end of the differential transmission line, and is located in the same plane as the ground plane. With reference to [8], we know that for a stripline-fed bilateral Vivaldi antenna, the length of the inner eurve, $L_{\mathrm{in}}$, and the opening rate of the inner curve, $B_{\mathrm{in}}$, have a predominant effect on the scan input impedanee of the array. The element width defines the $E$ and $H$-plane spacing and has a strong cffect on the frcquency at which the mismatch anomaly occurs. At present, no techniques are known to suppress this type of resonance, so that it inevitably fixes the upper limit of the usable bandwidth. However, to some extent, the resonance can be shifted up in frequency by a decrease of the aperture height or width of the elements. Furthermore, an increase in the ratio of the aperture height to the antenna width will produce an enlargement of the bandwidth, though a narrower scan pattern in the $H$-plane is to be expected when the aperture height is increased. As for the presently proposed differential antenna, the additional aperture length, $L_{\text {out }}$, and opening rate, $B_{\text {out }}$, of the outer curve are analyzed. The outer curve aims to realize a gradual transition from coplanar strip to slotline technology such as to achieve an ulırawideband antenna performance. Both the inner and the outer curves are described by the exponential (1) and (2). The slot and strip width have only a minor influence on the scan impedance, as opposed to the aforementioned parameters [8]; therefore these parameters are chosen to be invariant in this study. Note that two sets of axes are being used, one for the inner curve $\left(z_{1}-y_{1}\right)$, and another one for the outer one $\left(z_{2}-y_{2}\right)$.

The inner curve is described by

$$
\begin{aligned}
z_{1} & =A_{\text {in }} \cdot\left[\exp \left(B_{\text {in }} \cdot y_{1}\right)-1\right] \\
A_{\text {in }} & =W_{\text {in }} /\left[\exp \left(B_{\text {in }} \cdot L_{\text {in }}\right)-1\right] \\
\text { width } / 2-W_{\text {in }} & =4.5 \mathrm{~mm} .
\end{aligned}
$$

The outer curve is described by

$$
\begin{aligned}
z_{2}= & A_{\text {out }}\left[\operatorname{cxp}\left(B_{\text {out }} \cdot y_{2}\right)-1\right] \\
A_{\text {out }}= & W_{\text {out }} /\left[\operatorname{cxp}\left(B_{\text {out }} \cdot L_{\text {out }}\right)-1\right] \\
W_{\text {out }}= & {[\text { Width }- \text { Slot_width }] / 2 } \\
& - \text { Strip_width } \\
\text { Slot_width }= & 1.5 \mathrm{~mm} \\
\text { Strip_width }= & 4 \mathrm{~mm} \\
L_{\text {tot }}= & L_{\text {in }}+21 \mathrm{~mm} .
\end{aligned}
$$

The difference between hall the antenna width and hall the aperture width $\left(W_{\text {in }}\right)$ is fixed to $4.5 \mathrm{~mm}$ for assembly purposes. The $21 \mathrm{~mm}$ offset between the length of the inner curve $\left(L_{\text {in1 }}\right)$ and the total height of the antenna $\left(L_{\text {tot }}\right)$ is kept constant.

The initial geometrical parameters of the array are shown in Table I. For each of the following parametric analyses, only one of the parameters is swept, while fixing the other parameters in
TABLE I

InItlal, Grombtrical, ParambThes of lilk, Arkay

\begin{tabular}{|c|c|c|c|c|} 
Width & $I_{\text {in }}$ & $B_{\text {in }}$ & $I_{\text {out }}$ & $B_{\text {out }}$ \\
\hline $175 \mathrm{~mm}$ & $300 \mathrm{~mm}$ & $0.005 \mathrm{~mm}^{-1}$ & $60 \mathrm{~mm}$ & $0.08 \mathrm{~mm}^{-1}$ \\
\hline
\end{tabular}

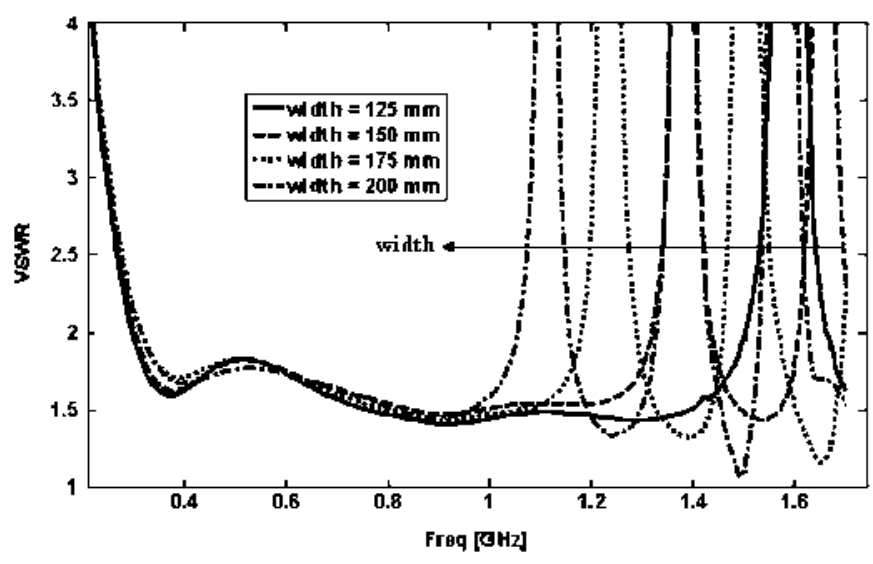

Fig. 4. VSWR at broadside over frequency for various antenna widths.

accordance to Table I. Furthermore, all results in this paper are obtained by exciting only one of the polarizations.

\section{A. Width of the Element}

The effect on the antenna input impedance, when reducing the width of the element, is visualized in Fig. 4. From this VSWR plot we conclude that the strong impedance anomaly in the upper part of the band is downshifted in frequency when the element width is enlarged, thereby limiting the usable band and giving rise to the strong mismatch visible in the VSWR (source-reference impedance is $150 \Omega$ ). This effect is also observed when the array is scanned in both the $E$ - and $H$-planes.

\section{$B$. Length of the Inner Curve $\left(L_{\mathrm{in}}\right)$}

A reduction of the taper length of the inner aperture will result in a narrowing of the impedance bandwidth while its center frequency shifts towards higher frequencies. The use of longer tapers lead to smaller fluctuations of the scan impedance, and a smaller reactance as well, particularly at lower frequencies, thereby improving the lower usable frequency (results not shown). However, the penalty is that the impedance anomalies in the upper part of the band move slightly down in frequency. A similar trend is observed when the array is scanned to $45^{\circ}$ in both principal planes.

In conclusion, the bandwidth of this type of arrays can be enlarged by increasing the length-width ratio of the inner taper of the antenna element, albeit to a certain extent.

\section{Opening Rate of the Inner Curve (Bin)}

The opening rate of the inner curve of the antenna is mainly affecting the matching level in the middle of the frequency band, though it also has a relatively significant effect on the lower limit of the usable bandwidth. Larger opening rates lead to stronger fluctuations in the scan impedance around their mean values. 


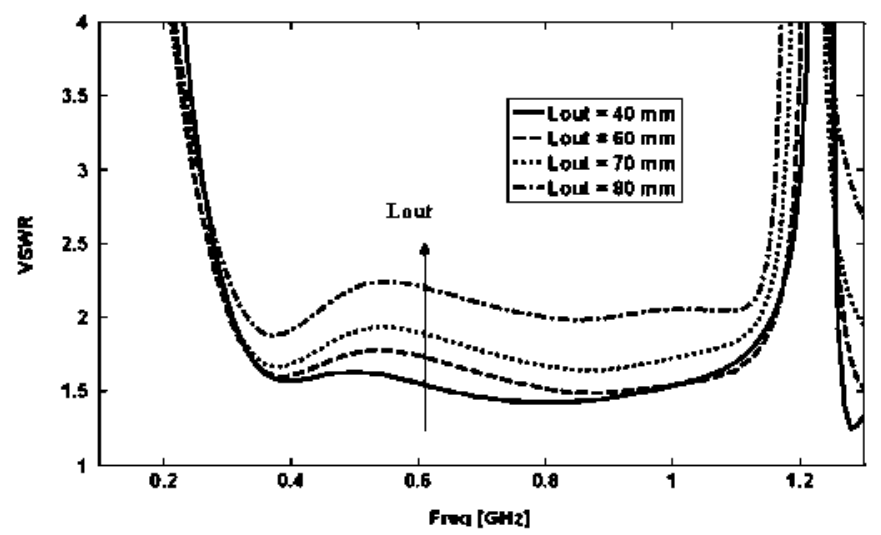

(a)

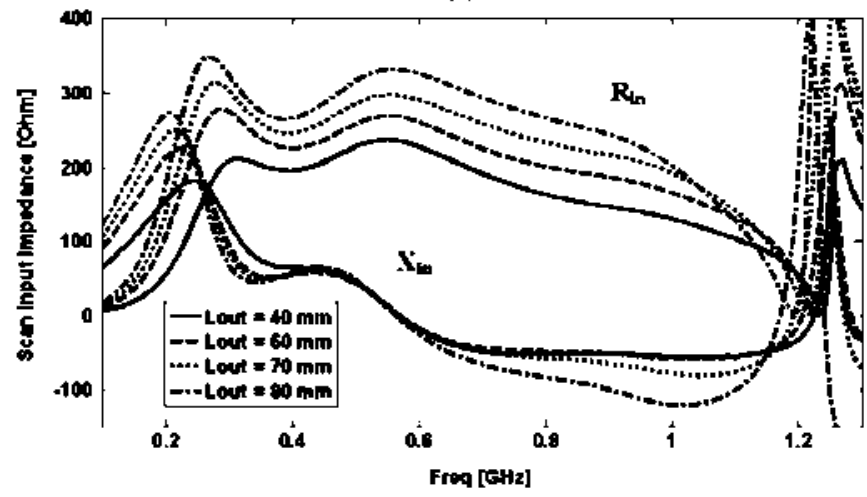

(b)

Fig. 5. A broadside scan over frequency for various antenna lengths of the outer curve: (a) VSWR and (b) the scan input impedance.

These mean values flatten-out for larger opening rates, especially at the upper part of the frequency band, and therefore result in better VSWRs. At the lowest end of the band, the VSWR increases with increasing $B_{\text {in }}$, so that it is necessary to find a compromise value for the opening rate of the inner curve. A similar behavior is observed when the array is scanned up to $45^{\circ}$ in both principal planes.

\section{Length of the Outer Curve ( $\left.L_{\text {out }}\right)$}

Results of scan impedance studies, and the occurrence of impedance anomalies, have been reported for the conventional stripline-fed bilateral Vivaldi antennas when the array scans offbroadside [6], [8]. To the author's best knowledge, no results have been reported for arrays of bunny-ear antennas where the effect of the outer curve on the scan impedance has been analyzed. It will be shown that several impedance effects are attributed to a specific design of the outer taper which need to be accounted for when designing and optimizing bunny-ear arrays.

The tapered outer eurve is an important part of the differential fecding system as its length controls the smoothness of the transition from coplanar strip to slotline technology and, in turn, influences the scan impedance of the antenna array elements. We can appreciate in Fig. 5(b) that an increase in taper length leads to an increase in the input resistance, whereas the input

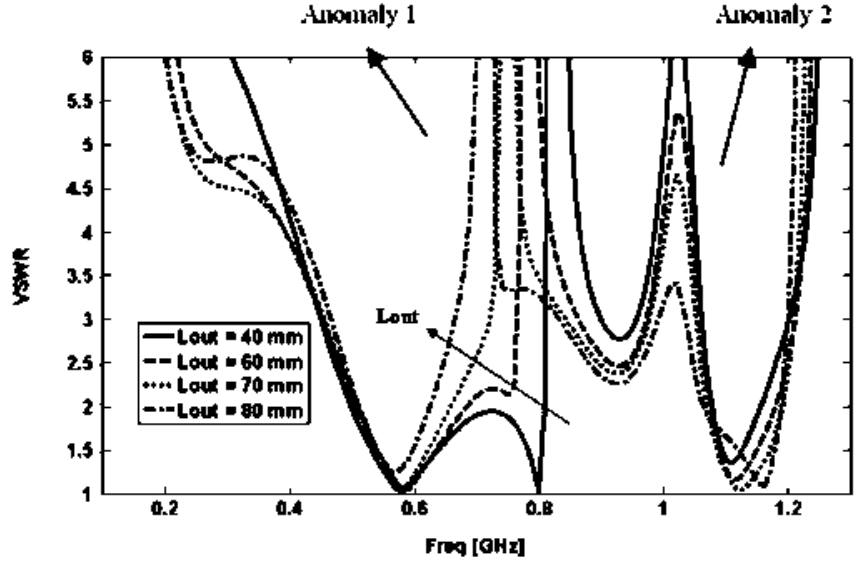

(a)

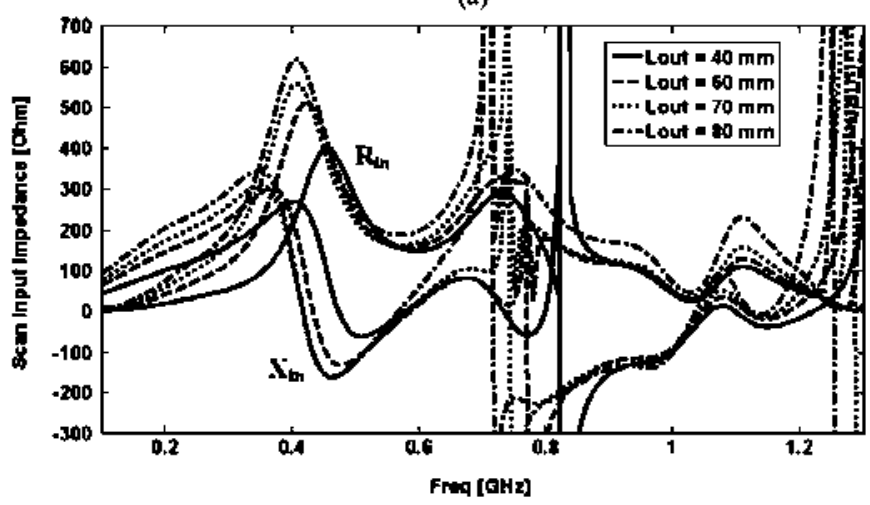

(b)

Fig. 6. A $45^{\circ}$ E-plane scan over frequency for various antenna lengths of the outer curve: (a) VSWR and (b) the scan input impedance.

reactance has virtually not changed, except at very low frequencies. Basically, only the matching level has changed as shown in Fig. 5(a), and the bandwidth has changed accordingly.

It is important to remark that for symmetric arrays of tapered slot antennas, typically only the $E$ - and $I I$-plane scans are of primary interest, since the $D$-plane scan impedance behaves, at least to first order, as a simple average of the $H$ - and $E$-plane scan performances [8].

Fig. 6 illustrates that when the array is scanned up to $45^{\circ}$ in the $E$-plane, an impedance anomaly [Anomaly 1 in Fig. 6(a)] appears within the band of interest and is strongly dependent on the length of the outer curve. It was found that the origin of this anomaly is related to the fact that the array can support a common-mode current. A more detailed explanation of its origin and suppression is given in Section III. So far, one can see how the length of the outer curve affects the position of the anomaly as it moves towards lower frequencies for larger taper heights.

The impedanec results are shown in Fig. 7 for the ease that the array is scanned to $45^{\circ}$ in the $H$-plane. An impedance resonanec appears in the upper part of the frequency band, thereby reducing the usable bandwidth. As in the ease of Anomaly 2 in the $E$-plane scan of Fig. 6(a), the location of this $H$-plane scan anomaly turns out to be virtually invariant for taper heights, as opposed to the case of Anomaly 1 in the $E$-plane scan of Fig. 6(a). Anomaly 2 in the high part of the band is due to the 


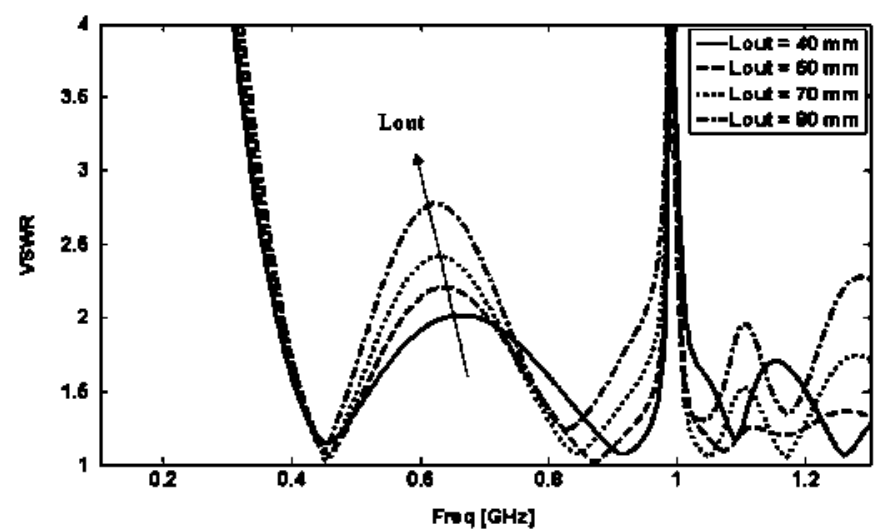

(a)

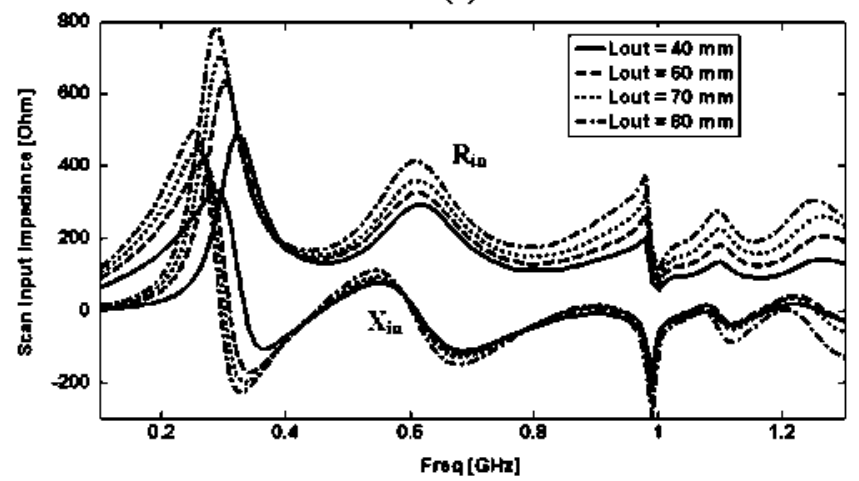

(b)

Fig. 7. A $45^{\circ} \mathrm{H}$-plane scan over frequency for various antenna lengths of the outer curve: (a) VSWR and (b) the scan input impedance.

appearance of the endfire grating lobe and is observed in the present unit cell computation because it corresponds to the frequency and angle at which a higher-order Floquet mode starts propagating.

Apart from the anomalous impedance effect, the trends observed in the scan impedances are very similar to the broadside-scanned array casc, i.c., the resistanec can be raised in-band by increasing the length of the outer taper, whereas the reactance remains almost the same. The mid-frequeney hump of the VSWR in Fig. 7(a) also increases with the height of the outer curve, and is in accordance with the increase in the input resistance.

\section{E. Opening Rate of the Outer Curve ( $B_{\text {out }}$ )}

The effects on the impedanee characteristies of the array are only weakly dependent on $B_{\text {out }}$. Furthemore, variations of the opening rate of the outer curve also have a minimal effect on the impedance anomalies, as opposed to a variation of $L_{\text {out }}$. The respective results are therefore not shown.

\section{SCAN Impedance ANOMALIES ASSOCiated to DIFFERENTIAL ARRAY TECHNOLOGY}

The presently proposed bunny-ear element exhibits a few $E$-plane scan impedance anomalies that are typically found for ocher Vivaldi elements as well [18], [19]. Apart from these types of resonances, a number of additional impedance anomalies exist that have been identified before and that are attributed to a particular realization of the TSA elements. Among them, (i)
TABLE II

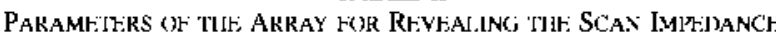
ANOMAIY

\begin{tabular}{|c|c|c|c|c|}
\hline Width & $L_{\text {in }}$ & $B_{\text {in }}$ & $L_{\text {unt }}$ & $B_{\text {sut }}$ \\
\hline $175 \mathrm{~mm}$ & $250 \mathrm{~mm}$ & $0.005 \mathrm{~mm}^{-1}$ & $73 \mathrm{~mm}$ & $0.07 \mathrm{~mm}^{-1}$ \\
\hline
\end{tabular}

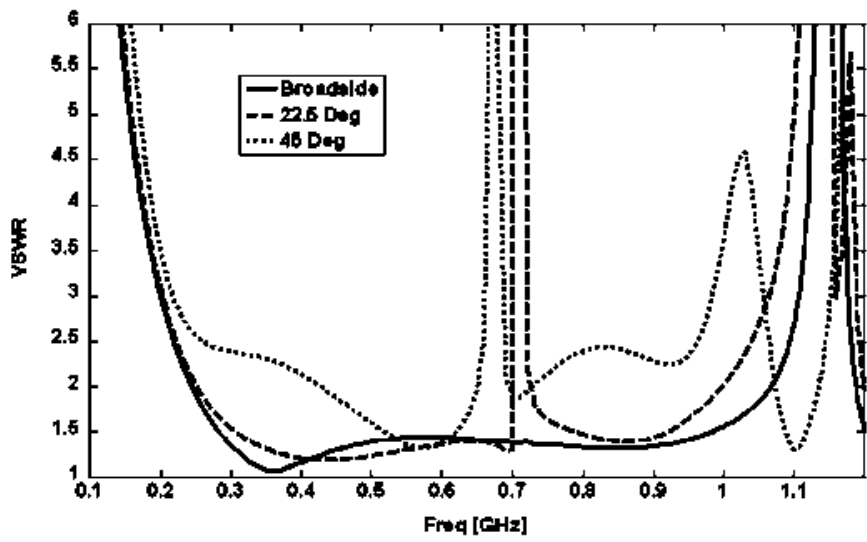

Fig. 8. VSWR for an $E$-plane scanned dual-polarized array.

the impedance resonance that appears when a surface excited wave travels along the facets of a substrate (printed TSAs), which then destructively interferes with the phasing of the array; (ii) the impedance resonances that appear due to gaps between disjoint antenna elements/tiles that tend to radiate, thereby severely disrupting the impedance characteristics [20]; (iii) the impedance resonances that occur for $H$-plane scanned bilateral TSAs, which arise due to a voltage difference between the bilateral fins of the TSAs, and that can be eliminated by plated-through vias in the elements [6]; or (iv) the impedanee resonance that arises in triangular grid arrays of phase-stecred linear-polarized TSA arrays [21]. The presently employed bunny-ear anlennas are dielectric-free, are electrically interconnected along their outer edges, and also unilateral and dual-polarized, so that the previously mentioned impedance resonances are not observed.

However, we identify here a type of impedance resonance which can specifically be attributed to differentially-excited arrays. To examine this type of scan impedanec anomaly, the geometrical parameters as listed in Table II are used.

The VSWR is shown in Fig. 8 for three different $E$-plane scans. Anomalous impedance effects occur for off-broadside scan angles in the middle of the band when exciting the antennas with a differential generator. We remark that these resonances disappear in the absence of a ground plane and shift down in frequency with an increase in scan angle. It is important to note that the position of the strong impedance anomalies in the upper part of the band also shift down for increasingly larger scan angles, in both the $E$-and $I I$-planes, though this effect is less pronounced as compared to the effect of increasing the element width, which reduees the operational bandwidth significantly.

In [19], similar anomalies appear in the middle of the band for $E$-plane scanned single-polarized arrays of bilateral TSAs, which are due to the presence of parallel plate modes in between the parallel strokes of TSA plates. This type of resonance was also found to $\propto$ cur for an $E$-plane scanned single-polarized 
array of bunny-ears (results omitted). As shown by Wunsch and Schaubert [22], these resonances can be suppressed by placing metallic cross walls at the locations where normally cross-polarized elements are placed.

In [23], one justifies the presence of an impedance anomaly that was found to occur because of the undesired radiation from the differential feed lines used to excite an array of dipole antennas. The behavior of these anomalies with frequency and scan angle are rather similar to the impedance perturbation presented in this paper.

In [24] a similar impedance anomaly for a planar array of symmetrical crossed dipoles has been identified and analyzed rigorously for the fields that are incident from grazing angle (in the $E$-plane). However, the polarization of the incident $E$-field vector is not the same as in the present work, where the array grid has been oriented perpendicular to the $E$-plane scans. Nonetheless, the anomalies seem to have a common origin with the one explained in this work, where we explain how currents couple to each other due to the presence of neighboring elements and how this leads to the undesired increase in the reflection coefficient.

In the work of Hay and O'Sullivan [11], an anomaly was found for a finite array of connected patches with features also observed in the present differentially-fed bunny-ear arrays ${ }^{1}$. Fig. 9 shows the topological equivalence between the two structures where $p$ is the geodesic path length along the edges of the conducting surfaces between the feed points of the elements of orthogonal polarizations. In [11] it is demonstrated that the frequency at which a differential-mode resonance occurs can approximately be predicted by the geodesic path length $p$ being half a wavelength. In the present study, the geodesic path length $p$ equals half a wavelength at $615 \mathrm{MHz}$, which is a reasonable prediction since the resonances are observed around 650-700 $\mathrm{MHz}$ (exact value depends on the scan angle $c f$. Fig. 8). Another property of this resonance is that it is characterized by anti-phase common-mode currents on the feed conductors of connected orthogonally polarized elements. In the following it is shown that this also occurs for the present structure.

By removing the ground plane, we retain only the two conductors (the two arms of the antenna) as well as the balanced feed lines. We observed that, for the present frequency range and for off-broadside scan angles, where the electrical symmetry is broken, only evanescent common-mode currents are supported along the differential feed lines.

However, in the presence of a third conductor, such as a ground plane, a common-mode signal can be supported by the feeding structure which may lead to severe differential-mode scan impedance resonances. From Fig. 8 one can observe for what frequencies impedance anomalies occur, and how these are dependent on the scan angle.

The surface current distribution on the antenna elements has been analyzed for a number of scan angles/frequencies, thereby revealing the presence of common-mode currents on the differential feed lines. It has been confirmed that both odd- and even- modes can exist on ground-plane-backed bunny-ear ar-

\footnotetext{
${ }^{1}$ When revising the manuscript, the author's attention was called to a recently published paper by Hay and O'Sullivan [11] where an equivalent situation is studied albeit for a different type of structure.
}

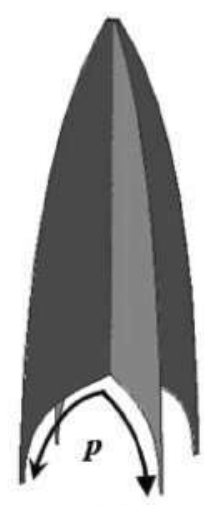

(a)

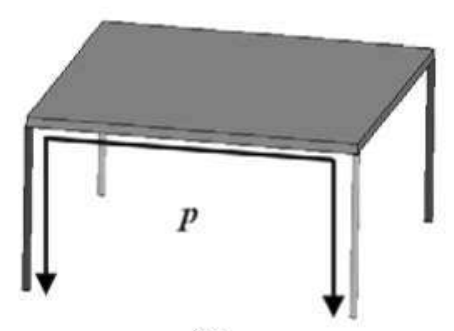

(b)
Fig. 9. Topological equivalence between the connecting surfaces of (a) the TSA elements and (b) the patches in [11]. Each drawing represents 4 half elements ( 2 per polarization). The geodesic path length along the conducting surfaces between the feed points of the elements of orthogonal polarizations is $p$.

rays, and these modes are excited differently, depending upon the frequency and scan angle. To analyze the various modes of the currents, we let a series of plane waves be incident to the open-circuited array while monitoring the induced current distributions. Fig. 10(a) shows the current distribution at $500 \mathrm{MHz}$, where one can clearly observe that the odd mode dominates over the even mode. The even-mode current distribution in Fig. 10(b) is strongly pronounced for an incident plane wave at $22.5^{\circ}$ at a frequency of $710 \mathrm{MHz}$, which is the frequency where a scan impedance anomaly occurs on transmit for the differentially-fed array scanned to $22.5^{\circ}$ in the $E$-plane. Fig. 10 therefore suggests that when the differential-mode scan impedance is at resonance, a common-mode current is supported by the structure, which may be excited strongly depending upon the type of excitation source used on transmit, or incident plane-wave field applied on receive. The presence of such a common-mode current at the anomalous frequency was also observed for an array of disconnected balanced antipodal Vivaldi antennas [25].

At this moment it is not clear how differential LNAs can be optimally noise matched to both types of scan impedances, given the fact that they are strongly related to each other. Despite an SKA-like station ( $>100,000$ elements) would largely behave as an infinite planar array [26], the common-mode anomaly in Fig. 8 could also appear at broadside, due to a slight asymmetry brought about by the finiteness of the array. An example is the case of a large array of disjoint subarrays, or a focal plane array with offset beams (non-symmetric excitations). Anomalies may also appear as observed by Schaubert, Craeye, and Boryssenko when introducing other types of asymmetries in the structure, e.g., [27], [28].

To mitigate the common-mode problem, i.e., to eliminate the resonance in the differential-mode scan impedance, it is essential to first analyze the propagation of the corresponding surface currents through the structure at resonance. Fig. 10(b) illustrates that the common-mode current is confined to the bottom region of the bunny-ear antennas instead of along the tapered slot as for the differential-mode current, and that the phase progression of the common-mode current starts at one arm of the antenna and ends in the arm of the orthogonally connected antenna. This specific problem is only present in dual-polarized arrays like the 


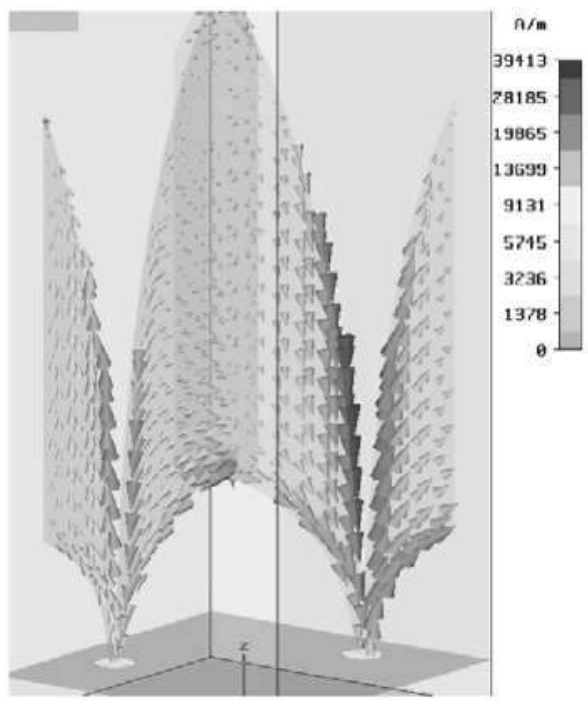

(a)

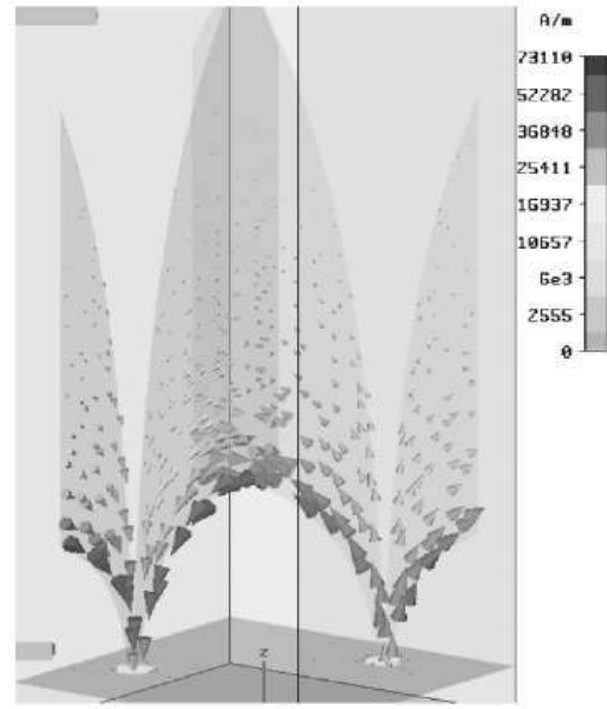

(b)

Fig, 10. Distribution of the surface currents for the unit cell at (a) $500 \mathrm{MHz}$ and (b) at the anomaly frequency with an incident plane wave at $22.5^{\circ}$.

ones in Fig. 2. Note that these characteristics were also observed for an array of connected patch antennas [11].

We will present a technique to avoid these impedance anomalies while keeping the orthogonally polarized elements in place. The comprise solution is based on using two resistors per element, each of them connected between one of the feeding lines and the GND plane, thereby dissipating a small part of the common-mode currents (see Fig. 11). Even though the proposed solution may seem very noisy for a radio astronomical application, it will be shown that the noise increment is only minor and therefore not of a big concern. The use of reactive elements has not shown to yield the desired change in the scan impedance characteristics.

Fig. 11 visualizes the two resistors that have been used to partly suppress the even mode current, thereby allowing the odd mode to dominate. The propagation path of the current reveals that it is sufficient to place the resistor in only one of the polarizations. However, in order to preserve the symmetry of the array we apply them to both polarizations.

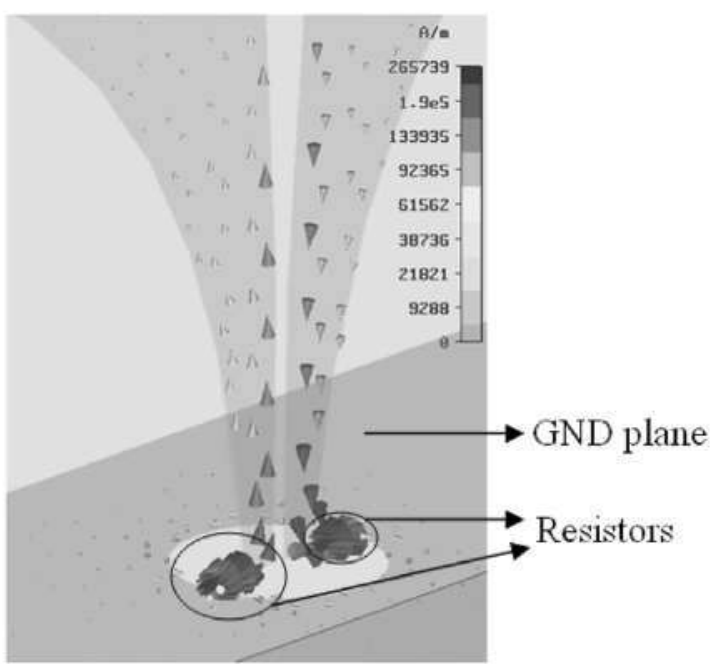

Fig. 11. Distribution of the surface currents for the unit cell after placing two resistors of $3 \mathrm{k} \Omega$ from each arm of the antenna to the ground plane. The current is shown at the anomalous frequency of Fig. 10(b).

A parametric study was undertaken to examine the antenna input impedance effect for various resistor values. For this purpose, the VSWR has been computed and plotted in Fig. 12 for a $45^{\circ} \mathrm{E}$-plane scan. Clearly, the differential VSWR increases when low-valued resistors are used since then the differential generator becomes almost short-circuited. The differential impedance match readily improves upon raising the resistor level by only tens of ohms. In fact, the even-mode currents keep sufficiently suppressed up to $3 \mathrm{k} \Omega$, while still a good overall matching level can be realized (VSWR $<2.5$ ). The differential scan impedance anomaly appears for resistor values larger than that, implying that the structure can support even-mode currents with significant amplitude. The case of raising the resistor values to infinity is equivalent to the case of removing the resistors (open circuit), so that the original anomalous differential VSWR is obtained allowing both the common-mode and the differential-mode currents to propagate. The common-mode current is stronger suppressed than the differential-mode current since the resistors are seen in series for the differential-mode excitation, whereas in parallel for the common-mode excitation (factor 4 difference, for DC). The best trade-off is reached when the resonance in the differential scan impedance becomes sufficiently suppressed while the overall impedance characteristics remain almost unaffected. This optimum resistor value was found to be near $3 \mathrm{k} \Omega$.

The differential VSWR for three different $E$-plane scans is presented in Fig. 13, both for $1 \mathrm{k} \Omega$ and $3 \mathrm{k} \Omega$ resistors. The $H$-plane scans are not shown, because these are only weakly dependent on the resistors. Note that for $3 \mathrm{k} \Omega$ resistors, the matching level in the band remains acceptable, even for large scan angles, and the results are very similar to those obtained for a resistor value of $1 \mathrm{k} \Omega$.

A thermal noise study of the proposed method is of interest when the lumped resistors are placed across the antenna conductors in front of the amplifying circuits ${ }^{2}$.

\footnotetext{
${ }^{2}$ The two loads in Fig. 11 may be realized by the input impedance of two single-ended LNAs which are used to form a differential balanced LNA, and like that, its noise temperature contribution is accounted for by the $S$-parameters and noise properties of the LNAs (antenna is regarded lossless).
} 


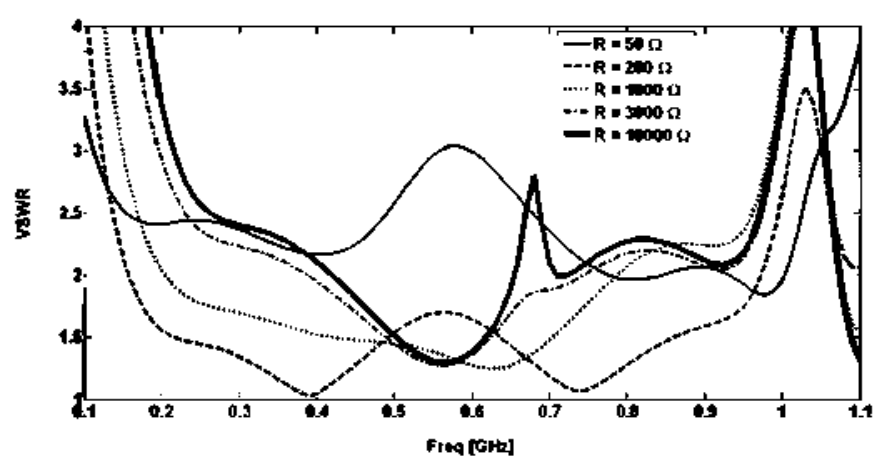

Fig. 12. VSWR for $\mathrm{a} 45^{\circ} \mathrm{E}$-plane scan for $\mathrm{R}=50 \Omega$ to $\mathrm{R}=10 \mathrm{k} 2$.

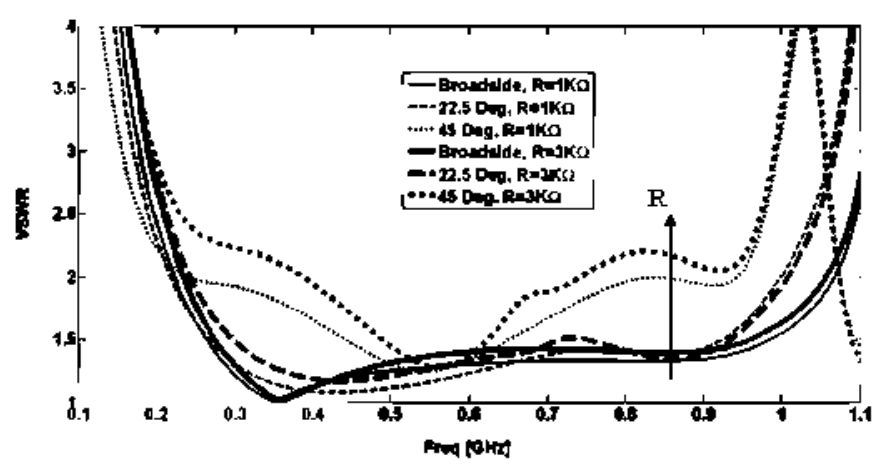

Fig. 13. VSWR for an $E$-plane scan, for $R=1 \mathrm{k} \Omega$ and $3 \mathrm{k} \Omega$.

The antenna impedance matching degrades by increasing the resistor values beyond a certain point as the effect of the lumped resistors decreases (Fig. 12), i.e., on transinil, a lower power is delivered to these resistors because of a low power transmission factor from the differential antenna port to the resistors. Likewise, on receive, only a sinall part of the thermal noise that has been generated by these resistors will be observed at the antenna port, due to this low transmission factor. In the following, we will account for the power absorption and associated noise temperature contribution through the radiation efficiency $\eta_{\mathrm{rad}}$ of the unit cell.

For receiving (phased-array) antennas, it is common to define the system sensitivity as the ratio of the effective aperture area $A_{\text {eff }}$ to the total system noise temperature $T_{s y s}$

$$
\frac{A_{\mathrm{efl}}}{T_{\mathrm{sys}}}=\frac{a b \cdot \cos (\theta) \cdot \eta_{\mathrm{rad}}}{\eta_{\mathrm{rad}} \cdot T_{\mathrm{sky}}+\left(1-\eta_{\mathrm{rad}}\right) \cdot T_{\mathrm{ambl}}+T_{\mathrm{LNA}}} .
$$

The above sensitivity formula, (5), is in accordance with (22) in [30], except that $\eta_{\mathrm{rad}}$ is here the radiation efficiency of the unit cell. It is important to inention that the sensitivity is herein referred to the inpnt of the LNA (ontput of the antenna). The effective area is defined through the antenna's available output power which is the available incident power to the unit cell area reduced by the radiation efficiency. By definition, we assume perfect polarization match and conjugate match tenminated array elements so that, in the absence of grating lobes, the effective area of a unit cell is given by the numerator of (5), where $\theta$ is

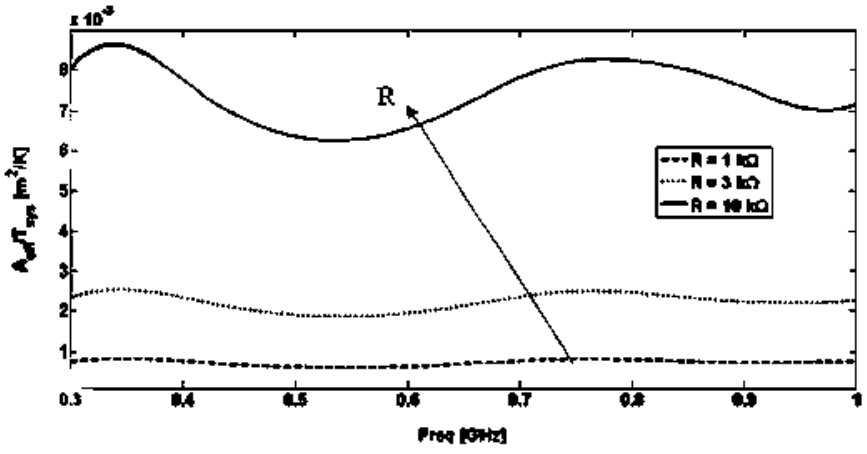

Fig. 14. System sensitivity per unit cell for a $45^{\circ} \mathrm{E}$-plane scan for three resistor values. The unit cell area $a b=30.6 \times 10^{-3} \mathrm{~m}^{2}$.

the direction of incidence measured from the nonnal to the array plane and $a b$ is the physical size of the unit cell (cf. [31]).

Apan from a power loss, the sensitivity also decreases due to an increase in the total system noise temperature caused by a decrease in the radiation efficiency. The temperature $T_{\text {amb }}$ is the physical antenna temperature, which equals (for non-cooled antennas) the typical ambient temperature of $290 \mathrm{~K}$, and $T_{\mathrm{L} . \mathrm{A}}$ is the noise temperature of the amplifier in the active scenario (with the source inpedance of the LNA equal to the scan inpedance), and $T_{\text {sky }}$ is the noise temperature contribution of the sky. For generality, both $T_{\text {sky }}$ and $T_{\mathrm{LNA}}$ are set to $0 \mathrm{~K}$ in order to be able to quantify the noise increase due to only the absorption losses of the antenna.

Fig. 14 demonstrates how the sensitivity per unit cell area increases when the value of the resistors increase. It is remarkable to observe that the sensitivity does not exhibit a resonance as e.g., observed in the VSWR port characteristics in Fig. 12, even not for $10 \mathrm{k} \Omega$ resistor values. This is due to the fact that the radiation efficiency itself is free of resonances since it only quantifies the relative dissipated power loss which may not change much when the port impedance is at resonance. On the contrary, $T_{\mathrm{LNA}}$ is expected to increase as a result of a noise misinatch to the anonalous differential source/antenna inpedance, but this effect is dependent on the specific LNA design which is not discussed in this paper.

In addition to the sensitivity curves, the noise temperature contributions for resistor values of $1 \mathrm{k} \Omega, 3 \mathrm{k} \Omega$ and $10 \mathrm{k} \Omega$ are presented in Fig. 15 for completeness. To be consistent with the noise temperature specifications used for the SKA, as well as with $Y$-factor measurements for antennas, it is common to refer the system noise tenperature in front of the antenna system (the sky), while the system sensitivity is a ratio and therefore independent on the chosen reference point. The corresponding noise teinperature forinula has been included in Fig. 15. We conclude that a compromise is necessary, i.e., increasing the resistor value leads to a lower noise telnperature (Fig. 15) but degrades the impedance match (Fig. 12). A good compromise has been found for a resistor value of $3 \mathrm{k} \Omega$, for which both a low noise temperature (Fig. 15) and a resonant-free $E$-plane scan impedance is realized which has a good matching level (Fig. 13). Fig. 15 demonstrates that the use of $3 \mathrm{k} \Omega$ resistors leads to a minor increment of the total system noise temperature, i.e., not more than 


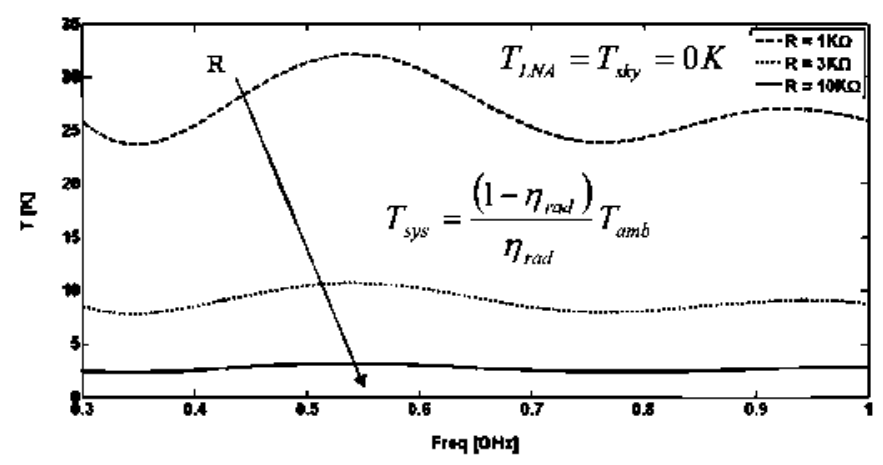

Fig. 15. System noise temperature for a $45^{\circ} E$-plane scan for three resistor values.

$10 \mathrm{~K}$, which is reasonable considering the $7 \mathrm{~K}(\sim 0.1 \mathrm{~dB}$ loss) that has been specified for the aperture array antennas in [13].

\section{DFsign EXAMPLF}

With the knowledge acquired in the parametric analysis of Seetion II, and by applying the impedance anomaly climination technique proposed in Section III, a dual-polarized array from $300 \mathrm{MHz}$ to $1 \mathrm{GHz}$ has been designed and optimized to eover a sean range up to $45^{\circ}$, thereby meeting the specifications of the SKA [26]. As we aim to minimize the cost, the element width is chosen as large as possible in order to minimize the number of amplifiers per square meter, while preventing in-band grating lobes from occurring.

The final geometrical parameters of the structure are shown in Table III, and the final results for several $E$-plane and $I I$-plane scans are plotted in Fig. 16. A resistor of $3 \mathrm{k} \Omega$ has been used to obtain these results. No $E$-plane impedanee anomalies are present and the VSWR is lower than 2.5 over the entire operational frequency band, as well as for all sean angles up to $45^{\circ}$.

\section{CONCLUSION AND FUTURE WORK}

A dual-polarized array of differentially-excited elements (bunny-ear antennas) has been analyzed through a parametric study in order to showcase the effect of various antenna element geometries on the scan impedance characteristics of an infinite-by-infinite array.

In addition, an $E$-plane differential-mode scan impedance anomaly has been identificd, which is caused by an even-mode that is supported by the differentially-fed antenna array in the presence of a third conductor, such as a ground plane. It has been shown that the impedance resonance can be suppressed by a (low loss) balancing-dissipation technique using a pair of resistors. The associated reduction in system sensitivity has been analyzed and shows that the increment of the corresponding antenna noise temperature is less than $10 \mathrm{~K}$ for $3 \mathrm{k} \Omega$ resistors at room temperature (290 K).

When passive resistors are used to prevent the differentialmode scan impedance anomaly from occurring, a compromise is necessary between: (i) the reduction in effective area as well as an increase in system noise temperature due to a reduced radiation efficiency, and; (ii) the realization of a resonant-free differential antenna impedance (source impedance) for achieving an optimal noise match between the differential LNAs and the

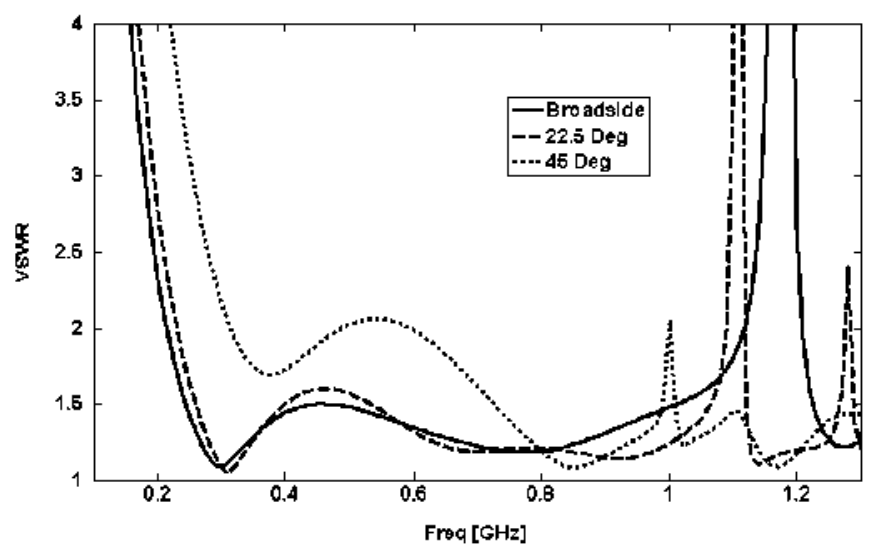

(a)

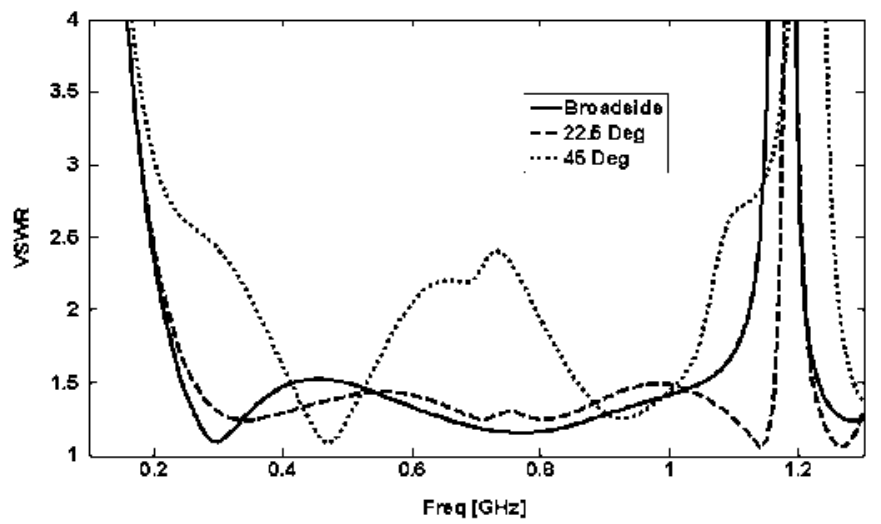

(b)

Fig. 16. Differential VSWR for various scans in the (a) $H$ plane, and (b) $E$ plane.

TABLE III

PARAMKTRRS OF THF OPTIMIZFID ARRAY

\begin{tabular}{|c|c|c|c|c|}
\hline Width & $L_{\text {in }}$ & $B_{\text {in }}$ & $L_{\text {uxt }}$ & $B_{\text {uut }}$ \\
\hline $168 \mathrm{~mm}$ & $350 \mathrm{~mm}$ & $0.008 \mathrm{~mm}^{-1}$ & $77 \mathrm{~mm}$ & $0.055 \mathrm{~mm}^{-1}$ \\
\hline
\end{tabular}

antenna elements. The latter depends on the specific LNA design which has not been discussed in this paper.

A final optimized antenna design has been presented meeting the specifications of the SKA project while keeping the potential manufacturing cost as low as possible. The operational frequency band ranges from $300 \mathrm{MHz}$ up to $1 \mathrm{GHz}$, the array is dual-polarized, and steerable up to $45^{\circ}$.

In future, we will elaborate upon the relation between the common-mode and the differential-mode scan impedances, as well as the optimal matching conditions that apply to differential antenna and LNA designs.

\section{ACKNOWLEDGMENT}

The authors would like to thank to the Astronomical Centre of Yebes, National Astronomical Observatory of Spain, for their comments and suggestions. They also want to thank Prof. D. $\mathrm{H}$. Schaubert for his comments, suggestions and time spent in private communications.

\section{REFERENCES}

[1] L. R. Lewis, M. Fasset, and J. Hunt, "A broad-band stripline array clement," in IEEE Int. Symp. Antennas Propag. Dig., 1974, pp. 335-337. 
[2] P. J. Gibson, "The Vivaldi aerial," in Proc. 9th Eur. Microw. Conf., Brighton, U.K., 1979, pp. 101-105.

[3] A. van Ardenne, H. Butcher, J. G. Bij de Vaate, A. J. Boonstra, J. D. Bregman, B. Woestenburg, K, van der Schaaf, P. N. Wilkinson, and M. A. Garrett, "The aperture array approach for the square kilometre array," [Online]. Available: www.skatelescope.org May 2003, White paper

[4] [Online]. Available: www.skatelescope.org

[5] "The European Concept for the SKA." [Online]. Available: www.skatelescope.org Jul. 2002, The Eur. SKA Consortium

[6] H. Holter, T. H. Chio, and D. H. Schaubert, "Experimental results of 144-element dual polarized endfire tapered-slot phased arrays," IEEE Trans. Antennas Propag., vol. 48, no. 11, pp. 1707-1718, Nov. 2000.

[7] R. Maaskant, M. Popova, and R. van de Brink, "Towards the design of a low cost wideband demonstrator tile for the SKA," presented at the Eur. Conf. on Antennas and Propag., Nice, France, 2006.

[8] T.-H. Chio and D. H. Schaubert, "Parameter study and design of wideband widescan dual-polarized tapered slot antenna arrays," IEEE Trans. Antennas Propag., vol. 48, no. 6, pp. 879-886, Jun. 2000.

[9] J. J. Lee and S. Livingston, "Wideband bunny-ear radiating element," in Proc. Antennas Propag. Society Int. Symp., 28 Jun.-2 Jul. 1993, vol. 3, pp. 1604-1607.

[10] S. G. Hay, J. D. O'Sullivan, J. S. Kot, C. Granet, A. Grancea, A. R. Forsyth, and D. H. Hayman, "Focal plane array development for ASKAP (Australian SKA pathfinder)," in Proc. 2nd Eur. Conf. on Antennas and Propag., Nov. 11-16, 2007, pp. 1-5.

[11] S. G. Hay and J. D. O'Sullivan, "Analysis of common-mode effects in a dual polarized planar connected-array antenna," Radio Science, RS6S04 2008.

[12] R. Maaskant, "Antenne-inrichting, antenne-array, samenstel voor het assembleren van een antenne-array en een elektronische inrichting omvattende een antenne," Dutch patent no. NL1034102.

[13] J. G. Bij De Vaate, L. Bakker, E. E. M. Woestenburg, R. H. Witvers, G. W. Kant, and W. Van Capellen, "Low cost low noise phased-array feeding systems for SKA pathfinders," presented at the ANTEM2009 Conf., Banff, Canada, Feb. 2009.

[14] M. Arts, R. Maaskant, E. de Lerea Acedo, and J. G. bij de Vaate, "Broadband differentially-fed tapered slot antenna array for radio astronomy applications," presented at the Eucap 3rd Eur. Conf. on Antennas and Propag., Berlin, Germany, Mar. 23-27, 2009.

[15] D. M. Pozar and D. Schaubert, "Scan blindness in infinite phased arrays of printed dipoles," IEEE Trans. Antennas Propag., vol. 32, no. 6, pp. 602-610, Jun. 1984

[16] J. P. R. Bayard, M. E. Cooley, and D. Schaubert, "Analysis of infinite array of printed dipoles on dielectric sheets perpendicular to a ground plane," IEEE Trans. Antennas Propag., vol. 39, no. 12, pp. 1722-1732, Dec. 1991.

[17] [Online]. Available: www.ansoft.com

[18] R. C. Hansen, Phased Arrays Antennas. New York: Wiley, 2001.

[19] D. Schaubert, "A class of E-plane scan blindnesses in single-polarized arrays of tapered-slot antennas with a ground plane," IEEE Trans. Antennas Propag., vol. 44, no. 7, pp. 954-959, Jul. 1996.

[20] D. H. Schaubert and A. O. Boryssenko, "Subarrays of Vivaldi antennas for very large apertures," in Proc. 34th Eur. Microw. Conf., Amsterdam, 2004, pp. 1533-1536.

[21] A. Ellgardt, "A scan blindness model for single-polarized tapered-slot arrays in triangular grids," IEEE Trans. Antennas Propag., vol. 56, no. 9, pp. 2937-2942, Sep. 2008.

[22] J. Wunsch and D. Schaubert, "Full and partial crosswalls between unit cells of endfire slotline arrays," IEEE Trans. Antennas Propag., vol. 48 , no. 6 , pp. 981-986, Jun. 2000.

[23] J.-P. R. Bayard, D. H. Schaubert, and M. E. Cooley, "E-plane scan performance of infinite arrays of dipoles on protruding dielectric substrates: Coplanar feed line and E-plane metallic wall effects," IEEE Trans. Antennas Propag., vol. 41, no. 6, pp. 837-841, Jun. 1993.

[24] E. L. Pelton and B. A. Munk, "Scattering from periodic arrays of crossed dipoles," IEEE Trans. Antennas Propag., vol. AP-27, pp. 323-330, 1979.

[25] D. Schaubert, S. Kasturi, M. W. Elsallal, and W. Van Cappellen, "Wide bandwidth Vivaldi antenna arrays-Some recent developments," presented at the EuCAP 2006, Nice, France, Nov, 6-10, 2006.

[26] R. T. Schilizzi et al., "Preliminary specifications for the square kilometre array," SKA Memo 100 Dec. 2007 [Online]. Available: http:// www.skatelescope.org/PDF/memos/100_Memo_Schilizzi.pdf

[27] M. W. Elsallal and D. H. Schaubert, "Electronically scanned arrays of dual-polarized, doubly-mirrored balanced antipodal Vivaldi antennas (DmBAVA) based on modular elements," in Proc. IEEE Antennas and Propag. Society Int. Symp., Jul. 9-14, 2006, pp. 887-890.

[28] C. Craeye, "Efficient simulation off finite wideband arrays-Reconciling finite and infinite-array approaches," presented at the Design of wideband receiving array systems SKADS MCCT technical workshop, Dwingeloo, The Netherlands, Nov. 26-30, 2007, Course Held as ASTRON Under the SKADS Framework

[29] E. García, E. De Lera, D. Segovia, and V. González, "Elimination of scan impedance anomalies in phased arrays," in Proc. IEEE Antennas and Propag. Society Int. Symp., San Diego, CA, Jul. 5-11, 2008, pp. $1-4$

[30] M. Ivashina, R. Maaskant, and B. Woestenburg, "Equivalent system representation to model the beam sensitivity of receiving antenna arrays," IEEE Antennas Wireless Propag. Lett., vol. 7, pp. 733-737, 2008.

[31] C. Craeye and M. Arts, "On the receiving cross section of an antenna in infinite linear and planar arrays," Radio Sci., vol. 39, no. RS2010, 2004.

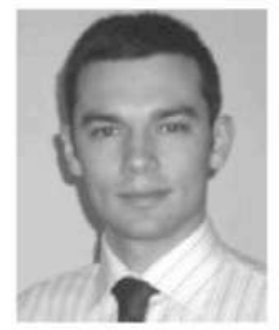

Eloy de Lera Acedo (S'05) was born in Spain in 1982. He received the Electrical Engineering degree (best graduate of the year distinction) from Carlos III University, Madrid, Spain, in 2005, where he is currently working toward the Ph.D. degree.

From 2006 to 2007, he worked at the Yebes Astronomical Center and stayed at the Netherlands Institute for Radio Astronomy (ASTRON) working in the design of antenna arrays for radio astronomy. Currently, he has a position in the Cavendish Laboratory, University of Cambridge, Cambridge, U.K. His main research interests include ultrawideband antenna arrays as well as radio astronomy receivers.

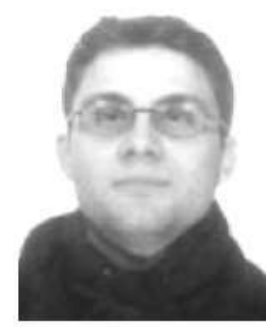

electromagnetics.
Luis Enrique García-Muñoz (M'99) received the Telecommunications Engineer degree and the Ph.D. degree in telecommunication from the Universidad Politécnica de Madrid, Madrid, Spain, in 1999 and 2003, respectively.

$\mathrm{He}$ is currently an Associate Professor with the Department of Signal Theory and Communications, Carlos III University, Madrid, Spain. His main research interests include radio-astronomy receivers, radiotelescopes, microstrip patch antennas and arrays, as well as periodic structures applied to

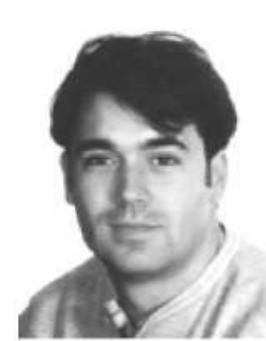

Vicente González-Posadas was born in Madrid. Spain, in 1968. He received the Ing. Técnico degree in radio-communication engineering from the Polytechnic University of Madrid (UPM), Madrid, Spain, in 1992, the M.S. degree in physics from the Universidad Nacional de Educación a Distancia (UNED), Madrid, Spain, in 1995, and the Ph.D. degree in telecommunication engineering from Carlos III University, Madrid, Spain, in 2001.

$\mathrm{He}$ is currently an Assistant Professor with the Technical Telecommunication School, Departamento de Ingeniería Audiovisual y Comunicaiones, UPM. He has authored or coauthored over 60 technical conference, letter, and journal papers. His research interest are related to active antennas, microstrip antennas, CRLH lines and metamaterials, and microwave technology. 


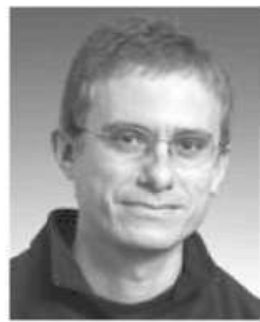

José Luis Vázquez-Roy was born in Madrid, Spain, in 1969. He received the Ingeniero de Telecomunicación and the Ph.D. degrees from the Universidad Politécnica de Madrid, Madrid, Spain, in 1993 and 1999, respectively.

In 1999, he joined the "Teoría de la Señal y Comunicaciones" Department, Universidad Carlos III de Madrid, where he is currently an Associate Professor. His research activities and interests include the development and characterization of planar antennas and circuits, the analysis of UWB antennas and the time domain computational electromagnetics.

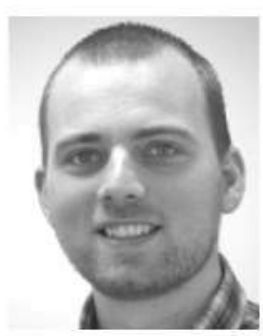

Rob Maaskant received the M.Sc. degree (cum laude) in electrical engineering from the Eindhoven University of Technology, The Netherlands, in 2003.

Since then, he has been an Antenna Research Engineer at the Netherlands Institute for Radio Astronomy (ASTRON) where his research is carried out in the framework of the Square Kilometre Array (SKA) Radio Telescope Project. He is currently working toward the Ph.D. degree in the field of numerically efficient integral-equation techniques for large finite antenna arrays. Besides, his research interest includes the characterization and design of antenna array receiving systems.

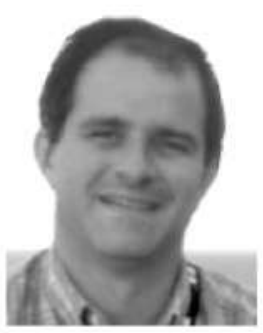

Daniel Segovia-Vargas (M'98) was born in Madrid, Spain, in 1968. He received the Telecommunication Engineering and $\mathrm{Ph} . \mathrm{D}$. degrees from the Polytechnic University of Madrid, Madrid, Spain, in 1993 and 1998 , respectively.

From 1993 to 1998, he was an Assistant Professor with Valladolid University. Since 1998, he has been an Associate Professor with Carlos III University, Madrid, Spain, where he is in charge of the microwaves and antenna courses. He has authored or coauthored over 60 technical conference, letters, and journal papers. His research interests are printed antennas and active radiators and arrays and smart antennas, left-handed (LH) metamaterials, and passive circuits. He has also been member of the European Projects Cost260, Cost284, and COST IC0603. 